\title{
Primer registro de Tylos niveus y nuevo reporte de Porcellionides pruinosus (Oniscidea: Tylidae y Porcellionidae) para Colombia
}

\author{
Yesenia M. Carpio-Díaz*, Carlos Mario López-Orozco, Yeison Herrera-Medina, \\ Gabriel R. Navas-S., Adriana Bermúdez
}

Universidad de Cartagena, Programa de Biología, Claustro San Pablo, Barrio Zaragocilla, Cartagena de Indias, Colombia

\begin{abstract}
Resumen
Se registra la presencia de Tylos niveus para Colombia y de Porcellionides pruinosus para la región Caribe colombiana. Los especímenes fueron recolectados en la Isla de Barú, Cartagena, departamento de Bolívar, Colombia. Se incluyen ilustraciones de los caracteres diagnósticos para ambas especies, y comentarios sobre su hábitat, morfología y distribución. (c) 2016. Acad. Colomb. Cienc. Ex. Fis. Nat.

Palabras clave: Isópodos terrestres; Neotrópico; Playa Blanca; Bolívar.
\end{abstract}

First record of Tylos niveus and new report of Porcellionides pruinosus (Oniscidea: Tylidae and Porcellionidae) for Colombia

\begin{abstract}
The presence of Tylos niveus and Porcellionides pruinosus is recorded from Colombia and the Colombian Caribbean respectively. The specimens were collected on the Baru Island, Cartagena, Bolivar department, Colombia. Illustrations of diagnostic characters, comments about habitat, morphology and distribution are included. (C) 2016. Acad. Colomb. Cienc. Ex. Fis. Nat.
\end{abstract}

Key words: Terrestrial isopods; Neotropics; Playa Blanca; Bolívar.

\section{Introducción}

Hasta abril de 2014 se habían descrito 3710 especies de isópodos del suborden Oniscidea (Sfenthourakis \& Taiti, 2015). Para Colombia, a pesar de ser un país megadiverso, solo se cuenta con registros de 37 especies (Richardson, 1914; Pearse, 1915; Vandel, 1972; López-Orozco, et al. 2014), 15 de ellas para la región Caribe (Pearse, 1915; Leistikow, 2001), lo cual es un número bajo en comparación con otros países de Suramérica como Brasil para el cual se conocen aproximadamente 161 (Campos-Filho, et al. 2014).

Los isópodos terrestres cumplen un papel esencial en los procesos de descomposición de la materia orgánica (Ŝpaldoňová \& Frouz, 2014; Abd El-Wakeil, 2015); han servido como organismos modelo de investigación en ecología (Sghaïer \& Charfi-Cheikhrouha, 2002; Hamaied \& Charfi-Cheikhrouha, 2004; Sfenthourakis, et al. 2005), reproducción (Araujo \& Bond-Buckup, 2005), fisiología (Lesěr, et al. 2008) y han sido utilizados como indicadores de impacto ambiental (Paoletti \& Hassall, 1999; Longo, et al. 2013).

El género Tylos Audouin, 1826 incluye especies generalmente supralitorales de playas tropicales y subtropicales de todo el mundo (Brown \& Odendaal, 1994). Cuenta con 21 especies descritas, de las cuales Tylos latreilli Audouin, 1826 ha sido reportada para Colombia (Pearse, 1915). El género Porcellionides Miers, 1878 está compuesto por especies con movimientos rápidos, gregarias y mésicas (Souza, et al. 2013). Este género posee 51 especies descritas, de las cuales Porcellionides pruinosus (Brandt, 1833) es conocida para el centro de Colombia. En este trabajo se registra la presencia de Tylos niveus (Budde-Lund, 1885) para Colombia y de Porcellionides pruinosus para la región Caribe colombiana, a partir de ejemplares recolectados en la Isla de Barú, departamento de Bolívar.

\section{Materiales y métodos}

Área de estudio: la isla de Barú se encuentra ubicada en el departamento de Bolívar, $18 \mathrm{Km}$ al sur de Cartagena, entre los $10^{\circ} 19^{\prime}$ y $10^{\circ} 18^{\prime}$ Norte, $75^{\circ} 42^{\prime}$ y $75^{\circ} 31^{\prime}$ Oeste (Figura 1). Abarca aproximadamente 7550 ha y es considerada como zona de amortiguamiento del Parque Nacional Natural Corales del Rosario y San Bernardo. La temperatura es de $27.7^{\circ} \mathrm{C}$, con valores mínimos de $21^{\circ} \mathrm{C}$ y máximos de $33^{\circ} \mathrm{C}$, y la humedad relativa oscila entre 80 y 85 \% (Valle, et al. 2011).

\footnotetext{
*Correspondencia:

Yesenia M. Carpio-Díaz, ycarpiod@unicartagena.edu.co

Recibido: 24 de febrero de 2016

Aceptado: 28 de junio de 2016
} 


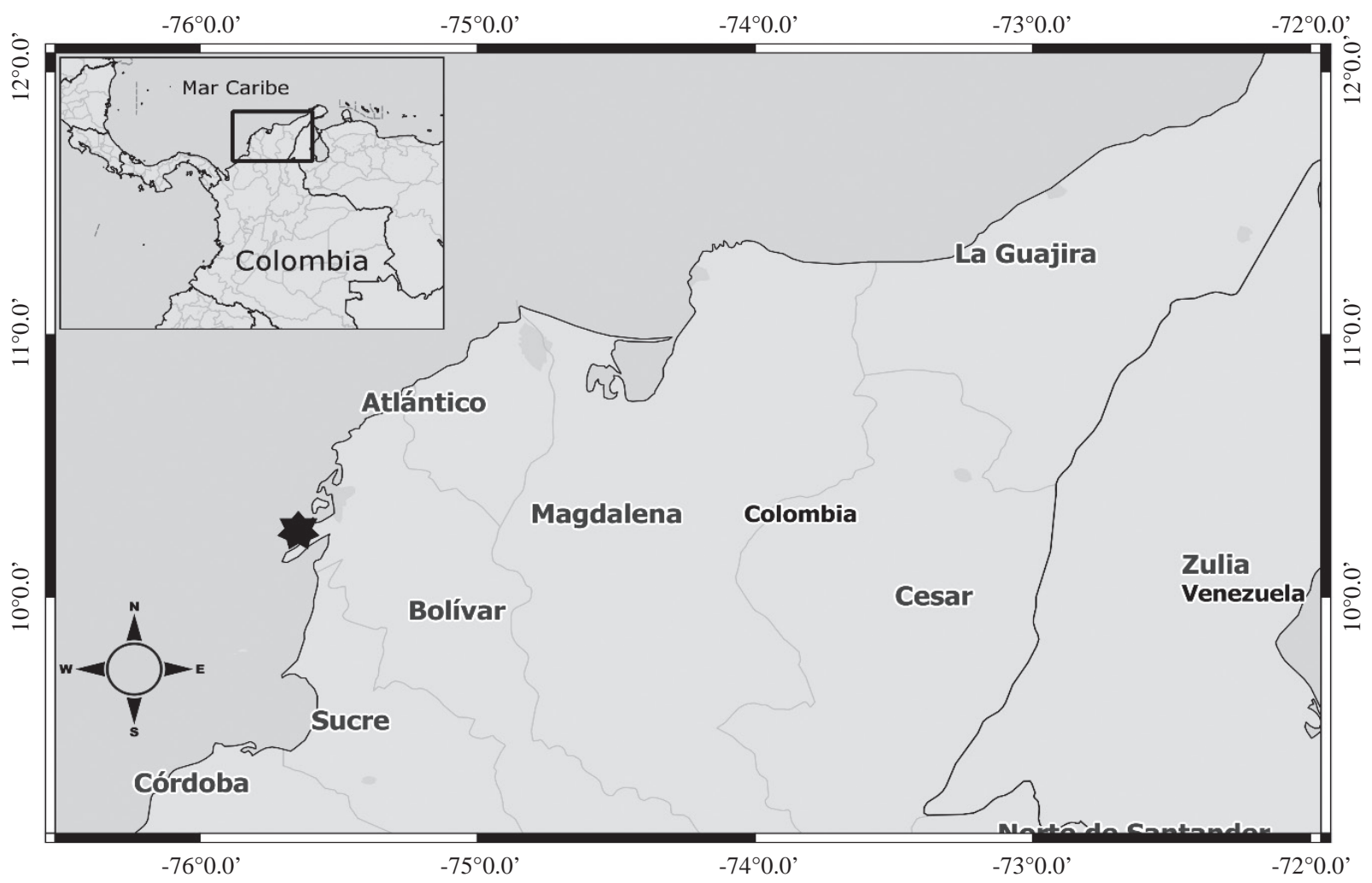

Figura 1. Localidad de muestreo de T. niveus y P. pruinosus, Colombia, departamento de Bolívar, Isla de Barú, Cartagena, $10^{\circ} 12^{\prime} 52.83^{\prime}$ Norte, 75³7’4.70”' Oeste.

Trabajo de campo y de laboratorio: los ejemplares fueron recolectados manualmente en Playa Blanca, Isla de Barú en el mes de agosto de 2015.

Los especímenes se preservaron en alcohol al 70\% y fueron identificados utilizando los trabajos de Boone (1934), Schultz (1970), Kensley \& Schotte (1989) y Souza, et al. (2013). Las ilustraciones se realizaron mediante el programa GIMP versión 2.8 siguiendo el método propuesto por Coleman (2006). El material analizado fue depositado en la Colección de Referencia del Programa de Biología de la Universidad de Cartagena (CUDC-CRU) y en la Colección de Crustáceos Isópodos del Instituto de Ciencias Naturales, Universidad Nacional de Colombia, Bogotá, Colombia (ICN-CI).

\section{Resultados y discusión}

\section{Porcellionidae Brandt, 1831}

Porcellionides Miers, 1877

Porcellionides pruinosus (Brandt, 1833)

Material examinado: Colombia. Bolívar: 2 q, $8 \mathrm{~mm}$ longitud máxima; 1 §, $9 \mathrm{~mm}$ longitud máxima, Cartagena, Isla de Barú, Playa Blanca, $10^{\circ} 12^{\prime}$ Norte, $75^{\circ} 37^{\prime}$ Oeste, 0 m, 8 Agos. 2015, C.M. López-Orozco y Yesenia M. CarpioDíaz. CUDC-CRU 4.

Diagnosis: $P$. pruinosus posee los epímeros IV-VII con una sombra de color naranja en la parte posterior (Figura 2B, sombra negra). Antenas alargadas, cuando están extendidas llegan hasta el pereonito IV; flagelo antenal compuesto de dos artículos, el primero aproximadamente el doble del segundo en longitud (Figura 2E). El exopodito y endopodito del pleópodo II como en la figura 2C. Según Souza, et al. (2013), $P$. pruinosus tienen una pigmentación de color marrón en la superficie, con parches brillantes, antes de preservados en alcohol. Pereonitos cubiertos por pequeños tubérculos (Figura 2A). Exopodito del pleópodo I del macho piriforme (Figura 2D); exopoditos de los pleópodos I-II con pulmones pleopodales. Urópodos y epímeros del pleón marcados con una sombra naranja; rama interna del urópodo estrecha distalmente, más o menos tres veces mayor en longitud a la rama interna (Figura 2A). Telson subtriangular, con ápice agudo (Figura 2B).

Distribución: especie cosmopólita, de origen mediterráneo. En Colombia ha sido registrada en Medellín (1547 msnm); en el Cafetal La Camelia, Angelópolis, Antioquia (1820 msnm); cerca del Nevado del Ruíz, Caldas (3700 msnm) (Richardson, 1914). Van Name (1936) menciona que existen especímenes de $P$. pruinosus reportados para San Andrés Islas en el Museo de América. Recientemente Martínez, et al. (2014), hallaron la especie en cultivos hortícolas en el departamento de Boyacá (2540-2750 msnm). En Suramérica ha sido registrada para Venezuela (Schmidt, 2001), Ecuador (Van Name, 1936), Brasil y Argentina (Souza, et al. 2013). 

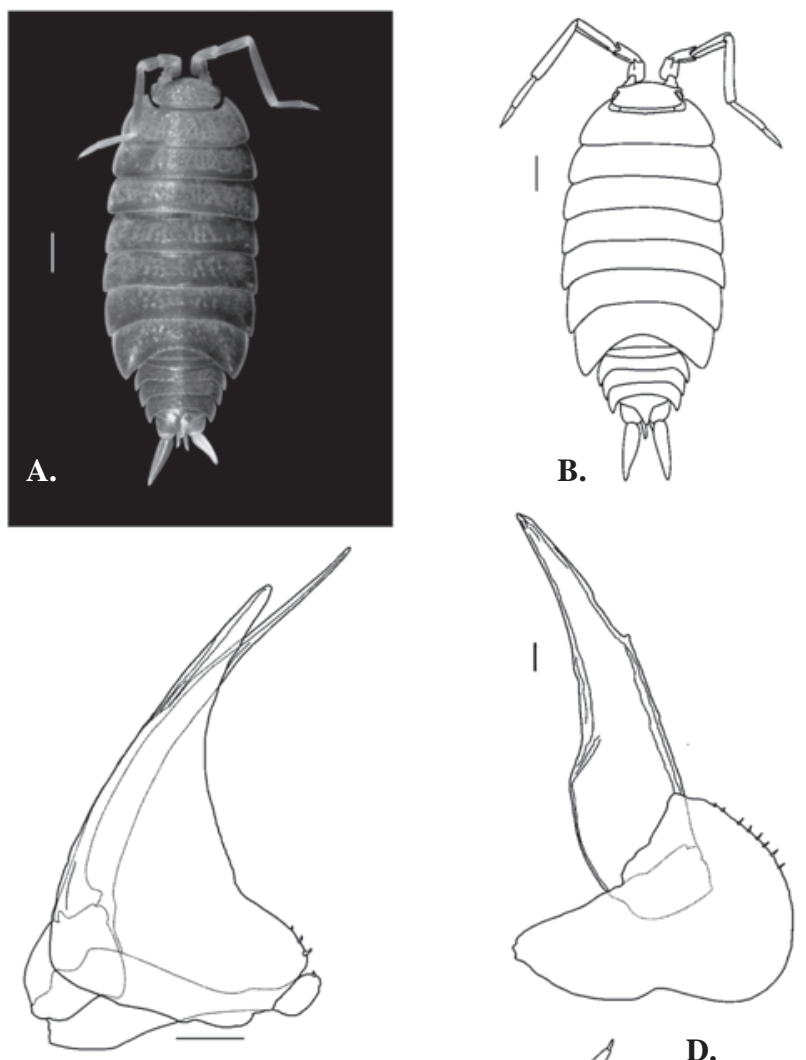

C.

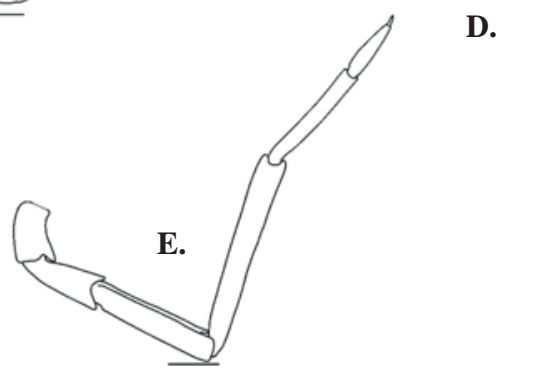

Figura 2. Porcellionides pruinosus. A y B. Vista dorsal; C. Exopodito y endopodito del pleópodo II; D. Exopodito y endopodito del pleópodo I; E. Antena. Barras de escala: Figs. A y B, 1 mm; Figs. C y D, 0.1 mm; Fig. E, 0.5 mm.

Comentarios: los especímenes fueron encontrados entre rocas y hojarasca de Coccoloba uvifera, a una distancia mínima de $10 \mathrm{~m}$ de la línea de costa.

Estudios genéticos entre poblaciones de $P$. pruinosus en Túnez revelaron poca variabilidad entre éstas (Achouri, et al. 2012); sin embargo, mediante el uso de análisis electroforéticos, reproductivos, distribucionales y morfológicos con poblaciones de Norte América, se describió una nueva especie: Porcellionides floria Garthwaite \& Sassaman, 1985, lo cual indica que existe una variabilidad entre poblaciones lo suficientemente separadas. Un análisis genético y/o morfológico entre las poblaciones de Suramérica podría revelar la existencia de nuevas especies o procesos de especiación, debido a las barreras climáticas y geográficas entre las zonas en las que se encuentran cada una de estas. Teniendo en cuenta la distribución altitudinal de esta especie en Colombia (0-3700 msnm), sería interesante realizar un estudio detallado de estas poblaciones con el fin de verificar si existen o no diferencias en cuanto a su genética y morfología.

\section{Tylidae Dana, 1852}

\section{Tylos Latreille, 1826}

Tylos niveus (Budde-Lund, 1885)

Material examinado: Colombia. Bolívar: 14 ภ, $12 \mathrm{~mm}$ longitud máxima; 23 + $12 \mathrm{~mm}$ longitud máxima, Cartagena, Isla de Barú, Playa Blanca, $10^{\circ} 12^{\prime}$ Norte, $75^{\circ} 37^{\prime}$ Oeste, 0 m, 8 Agos. 2015, Yeison Herrera-Medina y Ricardo Borja. CUDC-CRU 5. Colombia. Bolívar: 11 ภ, $10 \mathrm{~mm}$ longitud máxima; 21 + $11 \mathrm{~mm}$ longitud máxima, Cartagena, Isla de Barú, Playa Blanca, $10^{\circ} 12^{\prime}$ Norte, $75^{\circ} 37^{\prime}$ Oeste, 0 m, 8 Agos. 2015, C.M. López-Orozco y Yesenia M. Carpio-Díaz. ICN-CI-84.

Diagnosis: de acuerdo con Boone (1934), T. niveus presenta los ángulos posterolaterales de la cabeza redondeados; margen posterior recto (Figuras 3A y 3B). Segundo par de antenas con pedúnculo antenal compuesto de cinco segmentos; flagelo con cuatro segmentos (Figura 3D). El primer segmento torácico libre es el de mayor tamaño, con margen posterolateral redondeado (Figura 3C); segmentos II-VII de tamaño similar (Figuras 3A y 3B). El epímero del primer segmento es el de mayor tamaño, sin ningún tipo de sutura, con margen lateral proyectado hacia arriba, formando un surco ventral que se alarga posteriormente; epímero del segmento IV es el de menor tamaño; los epímeros II-IV con forma triangular; epímero V más o menos redondeado; epímeros VI-VII con forma cuadrangular (Figura 3C). Según Van Name (1936), Schultz (1970) y Kensley \& Schotte (1989), esta especie difiere del resto de las del género en que los filacomeros $\mathrm{V}$, izquierdo y derecho, son más o menos cuadrangulares y se encuentran en contacto en una larga línea medial (Figura 3F).

Distribución: esta especie ha sido considerada como típica de América. Distribuida en el Atlántico en México (Kensley \& Schotte, 1989); Florida, Cuba (Van Name, 1936); Bahamas, Tobago, Bonaire (Schultz, 1974); Dominica, Curazao, Islas Vírgenes, Belize, Venezuela (Kensley \& Schotte, 1989) y Brasil (Silva \& Alves, 2000).

Comentarios: en Florida se ha encontrado enterrada entre rocas y escombros a lo largo de la línea de costa (Schultz, 1974; Schultz \& Johnson, 1984). En Bonaire, se ha reportado entre la hojarasca de Avicenia sp. y Rizophora sp. junto con individuos de Ligia baudiniana H. Milne Edwards, 1840; en Curazao habita en la arena entre algas en descomposición, restos de corales con hojarasca de Laguncularia sp. y entre la hojarasca de Hippomane sp. (Schultz, 1974). En Brasil se ha encontrado viviendo entre gramíneas y entre la vegetación dominada por Ipomoea pes-caprae, que existe por encima de la porción de playa (Silva \& Alves, 2000). Todos los especímenes recolectados en Playa Blanca fueron encontrados durante el día, viviendo enterrados hasta 10 


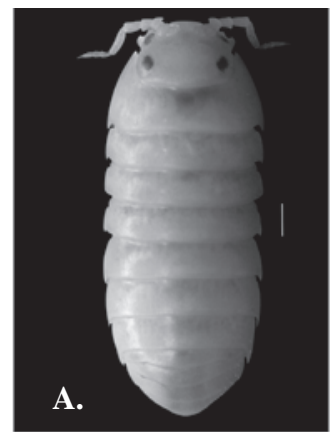

B.
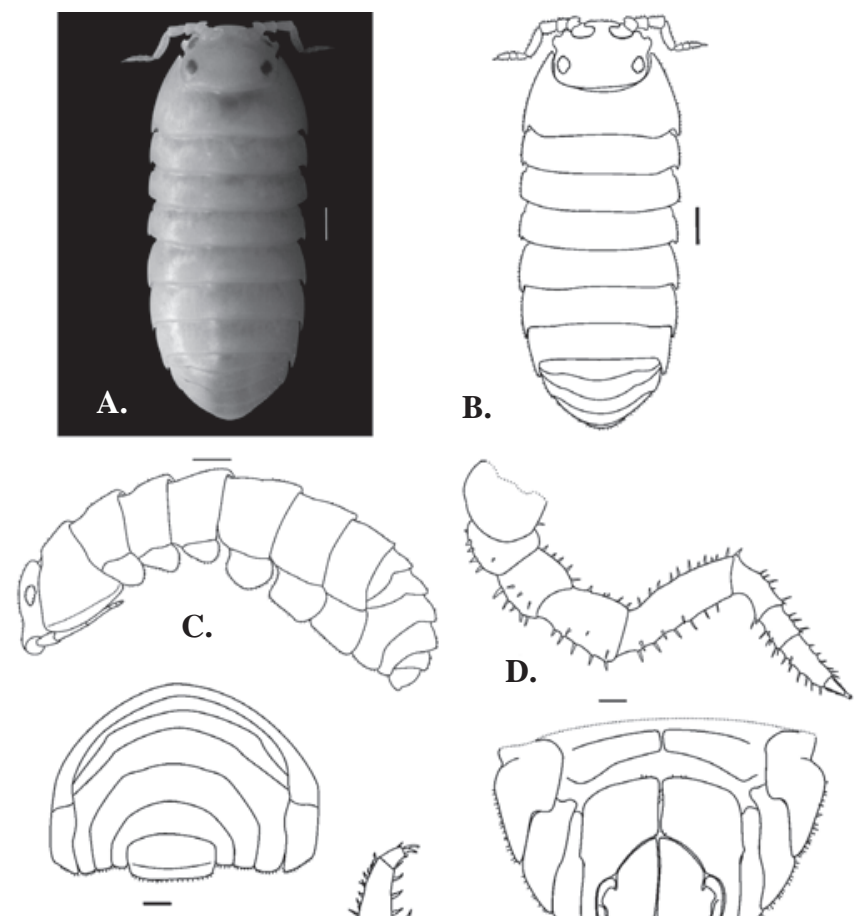

E.
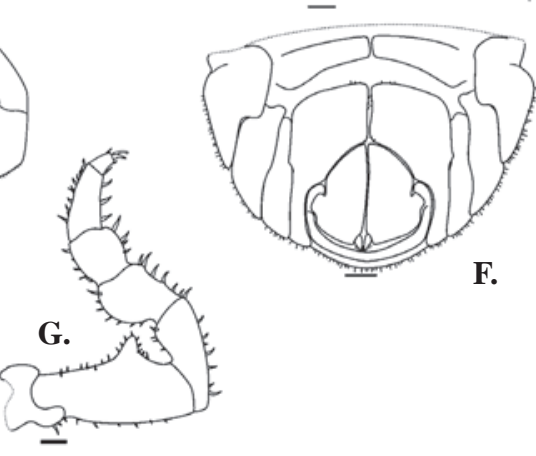

F.
Figura 3. Tylos niveus. A y B. Vista dorsal; C. Vista lateral; D. Antena; E. Telson en vista frontal; F. Telson en vista ventral; G. Pereiópodo I del macho. Barras de escala: Figs. A, B y C, 1 mm; Fig. D y G, 0.2 mm; Figs. E y F, 0.5 mm.

cm en la arena, entre hojarasca de Laguncularia racemosa y Coccoloba uvifera, a una distancia mínima de $10 \mathrm{~m}$ de la línea de costa.

Históricamente T. niveus ha sido identificada erróneamente como T. latreilli (Van Name, 1924; Schultz, 1972; Schultz \& Johnson, 1984). Arcangeli (1937) considera a $T$. niveus como una subespecie de T. latreilli. De acuerdo con lo propuesto por Van Name (1936), Schultz (1970) y Kensley \& Schotte (1989), estas dos especies pueden ser fácilmente identificadas teniendo en cuenta que en $T$. niveus los filacómeros $\mathrm{V}$ son más o menos cuadrangulares y se encuentran en contacto en una larga línea medial. Silva \& Alves (2000) realizaron la redescripción de T. niveus a partir de ejemplares recolectados en Santa Catarina, Brasil, mencionando que el filacómero $\mathrm{V}$ se encuentra yuxtapuesto en la línea media, en parte, al opuesto, además, en sus ilustraciones no se refleja dicho carácter (Ver Figura 4 en Silva \& Alves, 2000). Los especímenes de este estudio no presentan yuxtaposición entre los filacómeros izquierdo y derecho, lo cual concuerda con lo establecido por Van Name (1936), Schultz (1970) y Kensley \& Schotte (1989). Nuestros ejemplares presentan el basipodito del pereiópodo
I con proyección en forma de cono (Figura 3G) y un telson rectangular, con el margen posterior doblado hacia arriba (Figura 3E), caracteres diagnósticos que concuerdan con la redescripción de Silva \& Alves (2000). Por lo anterior, se requiere de un análisis detallado de las poblaciones de $T$. niveus en las diferentes localidades, con el fin de determinar si existe alguna variación y así establecer su identidad taxonómica. De igual manera, posiblemente un análisis comparativo entre las poblaciones de T. niveus y T. latreilli revelará la existencia o no de variaciones entre estas.

\section{Conclusiones}

Este es el primer registro de T. niveus para Colombia, y el segundo de un representante de este género para el país. Con el reporte de $P$. pruinosus para el departamento de Bolívar se confirma su presencia en el Caribe colombiano. Con estos dos registros se aumenta a tres el número de isópodos terrestres reportados para el departamento de Bolívar, y a 38 para Colombia, además, se amplía la distribución geográfica de estas dos especies en Suramérica y Colombia.

Es de resaltar la inexistencia de estudios de poblaciones de isópodos terrestres en Colombia, lo cual sería interesante realizar con estas dos poblaciones en el área de estudio, siendo ésta una zona de impacto antropogénico constante.

\section{Agradecimientos}

A Ricardo Borja Arrieta y Jaison Torres Pacheco por su colaboración en la colecta e identificación de los especímenes. A Erika Mejía, Daniela Ahumada, María Alejandra Padilla, Waldo Guerra, Elvis Padilla, Roy Gonzales y Hugo Vides por sus comentarios. Al profesor Ivanklin Soares Campos-Filho por su colaboración en la identificación y revisión del manuscrito. $\mathrm{Y}$ a los revisores por sus acertadas recomendaciones.

\section{Conflicto de intereses}

Los autores declaran no tener conflicto de intereses.

\section{Referencias}

Abd El-Wakeil, A.F. 2015. Effects of terrestrial isopods (Crustacea: Oniscidea) on leaf litter decomposition processes. The Journal of Basic \& Applied Zoology. 69: 10-16.

Achouri, M.S., Bouslama, M.F., Hamaïed, S. \& CharfiCheikhrouha, F. 2012. Genetic differentiation and gene flow of Porcellionides pruinosus (Crustacea: Isopoda: Oniscidea) in Tunisia. Journal of Natural History. doi:10.1 080/00222933.2012.707235.

Araujo, P.B. \& Bond-Buckup, G. 2005. Population structure and reproductive biology of Atlantoscia floridana (van Name, 1940. (Crustacea, Isopoda, Oniscidea) in southern Brazil. Acta Oecologica. 28: 289-298.

Arcangeli, A. 1937. Tylos latreillii Aud. et Sav., suoi biotipi, sua area di diffusione. Boll. dei Musei di Zool. e Anat. Comp. 46: 139-151.

Boone, L. 1934. New and rare Cuban and Haitian terrestrial Isopoda. Bulletin of the American Museum of Natural History. 66: 567-598. 
Brown, A.C. \& Odendaal, F.J. 1994. The Biology of Oniscid lsopoda of the Genus Tylos. Advances in Marine Biology. 30: 89-153.

Campos-Filho, I.S., Araujo, P.B., Bichuette, M.E., Trajano, E. \& Taiti, S. 2014. Terrestrial isopods (Crustacea: Isopoda: Oniscidea) from Brazilian caves. Zoological Journal of the Linnean Society. 172: 360-425.

Coleman, C.O. 2006. Substituting time-consuming pencil drawings in arthropod taxonomy using stacks of digital photographs. Zootaxa. 1360: 61-68.

Hamaied, S. \& Charfi-Cheikhrouha, F. 2004. Life cycle and Population dynamic of Armadillidium pelagicum Arcangeli, 1955 (Isopoda, Oniscidea) at Aouina. Comptes Rendus Biologies. 327: 343-352.

Kensley, B. \& Schotte, M. 1989. Guide to the marine isopod crustaceans of the Caribbean. Washington: Smithsonian Institution Press, 308 p.

Leistikow, A. 2001. A New Species of Terrestrial Isopoda from the Sierra Nevada de Santa Marta, Colombia (Crustacea: Oniscidea: Crinocheta). Studies on Neotropical Fauna and Environment. 36 (2): 151-158. doi: 10.1076/snfe.36.2. 151.2140.

Lesěr, V., Drobne, D., Vilhar, B., Kladnik, A., Žnidaršič, N. \& Štrus, J. 2008. Epithelial thickness and lipid droplets in the hepatopancreas of Porcellio scaber (Crustacea: Isopoda) in different physiological conditions. Zoology. 111: 419-432.

Longo G., Trovato, M., Mazzei, V., Ferrante, M. \& Conti, G.O. 2013. Ligia italica (Isopoda, Oniscidea) as Bioindicator of Mercury Pollution of Marine Rocky Coasts. PLOS ONE, 8 (3), e58548. doi:10.1371/journal.pone.0058548.

López-Orozco, C.M., Bermúdez, A. \& Navas, G. R. 2014. Primer registro de Ligia baudiniana (Crustacea: Isopoda: Oniscidea) para el Caribe Colombiano. Boletín de Investigaciones Marinas y Costeras. 43 (1): 195-200.

Martínez, J.W., Pérez, D.F. \& Espíndola, C.C. 2014. Caracterización de isópodos terrestres (Crustacea: Isopoda) y su impacto en cultivos hortícolas de Boyacá. Revista de Ciencias Agrícolas. 31 (1): 55-64.

Paoletti, M.G. \& Hassall, M. 1999. Woodlice (Isopoda: Oniscidea): their potential for assessing sustainability and use as bioindicators. Agriculture, Ecosystems and Environment. 74: 157-165.

Pearse, A. 1915. An account of the Crustacea collected by the Walker Expedition to Santa Marta, Colombia. Proceedings of the United States national Museum. 49: 531-556.

Richardson, H. 1914. Terrestrial Isopods of Colombia. In: O. Fuhrmann et Eug. Mayor. Voyage d'exploration scientifique en Colombie. Mémoires de la Société des Sciences Naturelles Neuchatel. 5: 29-32.

Schmidt, C. 2001. Lista preliminar de los isópodos terrestres (Crustacea, Isopoda, Oniscidea) de Venezuela. Boletín de la Sociedad Venezolana de Espeleología. 35: 1-12.
Schultz, G. 1970. A review of the species of the genus Tylos Latreille from the new world (Isopoda. Oniscidea). Crustaceana. 19: 297-305.

Schultz, G. 1972. Ecology and systematics of terrestrial isopod Crustaceans from Bermuda. Crustaceana Supplement. 3: 79-99.

Schultz, G. 1974. Terrestrial Isopod Crustaceans (Oniscoidea) mainly from the West Indies and adjacent regions: 1 . Tylos and Ligia. Studies on the fauna of Curaçao and other Caribbean islands. 149: 162-173.

Schultz, G. \& Johnson, C. 1984. Terrestrial isopod crustaceans from Florida (Oniscoidea). Tylidae, Ligiidae, Halophilosciidae, Philosciidae and Rhyscotidae. Journal of Crustacean Biology. 4 (1): 154-171.

Sfenthourakis, S. \& Taiti, S. 2015. Patterns of taxonomic diversity among terrestrial isopods. In: S. Taiti, E. Hornung, J. Štrus \& D. Bouchon (Eds). Trends in Terrestrial Isopod Biology. ZooKeys, 515: 13-25. doi: 10.3897/zookeys.515.9332.

Sfenthourakis, S., Anastasiou, I. \& Strutenschi, T. 2005. Altitudinal terrestrial isopod diversity. European Journal of Soil Biology. 41: 91-98.

Sghaïer, M. \& Charfi-Cheikhrouha, F. 2002. Biologie et dynamique de population de Porcellionides sexfasciatus (Crustacea, Isopoda, Oniscidea). Comptes Rendus Biologies. 325: 605-616.

Silva, J. \& Alves, E. 2000. Tylos niveus Budde-Lund, 1885 (Crustacea: Isopoda: Oniscidea: Tylidae): redescrição e nova ocorrência para a praia de Taquaras, Santa Catarina, Brasil. Acta Biológica Paranaense. 29 (1, 2, 3, 4): 265-285.

Souza, L.L., Azevedo, H., Vargas, A.B., Senna, A.R. \& Souza, L. A. 2013. First record of Porcellionides pruinosus (Brandt, 1833) (Oniscidea: Porcellionidae) from Trindade Island, off Espírito Santo state coast, Brazil. Boletim do Museu de Biologia Mello Leitão. 32: 71-78.

Ŝpaldoňová, A. \& Frouz, J. 2014. The role of Armadillidium vulgare (Isopoda: Oniscidea) in litter decomposition and soil organic matter stabilization. Applied Soil Ecology. 83: 186-192.

Valle, A.G., Osorno-Arango, A.M. \& Gil-Agudelo, D.L. 2011. Estructura y regeneración del bosque de manglar de la ciénaga de Cholón, Isla Barú, Parque Nacional Natural Corales del Rosario y San Bernardo, Caribe colombiano. Boletín de Investigaciones Marinas y Costeras. 40 (1): $115-130$

Van Name W.G. 1924. Isopods from the Williams Galapagos Expedition. Zoologica. 5: 181-210.

Van Name, W.G. 1936. The American land and fresh-water isopod Crustacea. Bulletin of the American Museum of Natural History. 71: 1-535.

Vandel, A. 1972. Les isopodes terrestres de la Colombie. Studies on Neotropical Fauna. 7 (2): 147-172. 